\title{
VIOLÊNCIA AUTOINFLIGIDA E ANOS POTENCIAIS DE VIDA PERDIDOS EM MINAS GERAIS, BRASIL
}

\author{
Fernanda Carolina Camargo ${ }^{1}$, Helena Hemiko Iwamoto ${ }^{2}$, Lorena Peres de Oliveira ${ }^{3}$, Renata Cobo de Oliveira
}

\footnotetext{
${ }^{1}$ Mestre em Atenção à Saúde, Programa de Pós-Graduação em Atenção à Saúde pela Universidade Federal do Triangulo Mineiro (UFTM). Minas Gerais, Brasil. E-mail: fernandaccamargo@yahoo.com.br

${ }^{2}$ Doutora em Enfermagem Fundamental. Professora Adjunto do curso de Graduação em Enfermagem da UFTM. Minas Gerais, Brasil. E-mail: helena.iwamoto@gmail.com

${ }^{3}$ Mestranda em Atenção à Saúde da UFTM. Minas Gerais, Brasil. E-mail: lorenaenfer@gmail.com

${ }^{4}$ Mestranda em Atenção à Saúde da UFTM. Minas Gerais, Brasil. E-mail: renatacobo@hotmail.com
}

RESUMO: Os dados mundiais evidenciam que, aproximadamente, um milhão de pessoas cometeram violência autoinfligida, em 2000, e as consequências têm sido alarmantes. Uma pessoa morre por esse motivo a cada 40 segundos, e outra atenta contra a própria vida a cada três segundos. Estudo exploratório, objetivando a análise epidemiológica de mortalidade por violência autoinfligida do Estado de Minas Gerais, por meio do cálculo do Coeficiente de Mortalidade Padronizado e Anos Potenciais de Vida Perdidos. A mortalidade, no período de 1996 a 2007, foi 4,29 óbitos por 100.000 habitantes, sendo superior nos homens $(6,90)$ em relação às mulheres $(1,73)$. A faixa etária de 40 a 59 anos atingiu mortalidade de 7,0 óbitos por 100.000 habitantes. Em relação aos anos de vida perdidos a média da idade de óbitos manteve-se em torno dos 40 anos, evidenciando a perda do potencial social do sujeito acometido pela violência autoinfligida.

DESCRITORES: Violência. Suicídio. Estudos epidemiológicos.

\section{SELF-INFLICTED VIOLENCE AND YEARS OF POTENTIAL LIFE LOST IN MINAS GERAIS, BRAZIL}

\begin{abstract}
Worldwide data shows that approximately one million people committed self inflicted violence in 2000 and that the consequences have been alarming. One person dies for this reason every 40 seconds, with another attempting to take his/her life every three seconds. This exploratory study targeting the epidemiological analysis of mortality by self-inflicted violence in the state of Minas Gerais, Brazil, through calculating the Standardized Mortality Coefficient and Potential Years of Life Lost. Mortality calculated from 1996 to 2007 was 4.29 deaths per 100,000 inhabitants, with men (6.9) outpacing women (1.73). The age range of 40 to 59 years reached a mortality coefficient of 7.0 deaths per 100,000 residents. In relation to the distribution of years of life lost, the average age of those who died remained around 40 years, focusing on the loss of the potential social subject taken by self-inflicted violence.
\end{abstract}

DESCRIPTORS: Violence. Suicide. Epidemiologic studies.

\section{LA VIOLENCIA AUTOINFLIGIDA Y LOS AÑOS POTENCIALES DE VIDA PERDIDOS EN MINAS GERAIS, BRASIL}

RESUMEN: Estadísticas mundiales demuestran que aproximadamente un millón de personas han cometido violencia autoinfligida en 2000, y las consecuencias han sido alarmantes. Una persona muere por esta razón a cada 40 segundos y otra ataca su propia vida a cada tres segundos. Es un estudio exploratorio destinado al análisis epidemiológico de la mortalidad por violencia autoinfligida en el Estado de Minas Gerais, a través del cálculo de la tasa de mortalidad estandarizada y los años potenciales de vida perdidos. La tasa de mortalidad desde 1996 hasta 2007 fue de 4,29 muertes por cada 100.000 habitantes, superior en los hombres $(6,9)$ que en las mujeres $(1,73)$. La población de 40 a 59 años alcanzó a una tasa de mortalidad de 7,0 muertes para cada 100.000 habitantes. En relación con los años de vida perdidos, la edad promedio de muertes se mantuvo en alrededor de 40 años, lo que demuestra la pérdida de potencial social de los sujetos afectados por la violencia autoinfligida.

DESCRIPTORES: Violencia. Suicidio. Estudios epidemiológicos. Enfermería en salud pública 


\section{INTRODUÇÃO}

O suicídio é um importante problema de saúde pública e tem alcançado caráter endêmico. Seus impactos, para o setor saúde, não contabilizam apenas as mortes provocadas por essa violência autoinfligida. Para esse segmento convergem os danos, as lesões e os traumas relacionados. ${ }^{1}$

Dados mundiais apontam que, aproximadamente, um milhão de pessoas cometeu suicídio, na década de 1990, e as consequências têm sido alarmantes. Uma pessoa morre por esse motivo a cada 40 segundos, e outra atenta contra a própria vida a cada três segundos. Para cada violência autoinfligida acometida, existem pelo menos dez tentativas. Para cada tentativa registrada, ocorrem outras quatro desconhecidas. Dessa maneira, a violência autoinfligida apresenta danos imensuráveis com impacto psicológico, social e financeiro, em pelo menos outras seis pessoas. ${ }^{2}$

No Brasil, em 2000, a violência autoinfligida foi responsável por 6.778 óbitos, que corresponderam a $5,70 \%$ do total de mortes, com taxa de mortalidade igual a 4,0/100 mil habitantes. Ressalta-se que, entre os homens a taxa de mortalidade foi de 5,0 óbitos e, para as mulheres, de 1,6 óbitos/100 mil habitantes. ${ }^{3}$

Vale ressaltar que a definição clássica de violência autoinfligida apresenta-se como: "todo caso de morte que resulte, direta ou indiretamente de um ato positivo ou negativo, praticado pela própria vítima, sabedora de que poderia produzir esse resultado". 4:16 Assim, violência autoinfligida é um ato consciente e autodestrutivo, que pode ser compreendido como uma enfermidade multidimensional. Além do mais, estudos recentes relacionam diversos fatores predisponentes à violência autoinfligida, classificados como proximais facilitadores da agressão, como a presença de uma arma de fogo no domicílio, ou distais, como base estruturante a tal comportamento, que, juntos, traduzem o cenário da violência autoinfligida. ${ }^{5-6}$

Observa-se que relevantes situações presente no cotidiano de vida, atual, potencializam o comportamento da violência autoinfligida. Dentre eles podem ser apontados o aumento da prevalência de transtornos depressivos; o crescimento do uso abusivo de substâncias psicoativas; as mudanças no padrão psicobiológico como: a diminuição na data de início da puberdade, o elevado número de agentes estressores, a maior aceitação social de comportamentos e modelos de violência autoinfligida. ${ }^{6-9}$

Nesse sentido, as mortes resultantes da violência autoinfligida devem ser destacadas, espe- cialmente, ao se considerar seus impactos sociais e familiares. ${ }^{8-10}$ Para cada violência autoinfligida há, em média, cinco ou seis pessoas próximas ao falecido que sofrem consequências emocionais, sociais e econômicas, resultando em 1,4\% do ônus global ocasionado por doenças. ${ }^{2}$

Não se pode deixar de levar em consideração que "a morte, quando ocorre numa etapa da vida de alta criatividade e produtividade, não só pune o próprio indivíduo e o grupo que lhe é próximo, mas também priva a coletividade de seu potencial econômico e intelectual" ${ }^{11: 189} \mathrm{E}$, ainda, essas ocorrências são permeadas por aspectos subjetivos e culturais e por essa razão, as abordagens desse agravo pelos profissionais de saúde devem se pautar na construção de proposições e intervenções qualificadas para o seu controle, levando-se em consideração a complexidade e multiplicidade desta temática. ${ }^{12}$

Na prática cotidiana em saúde, cabe aos profissionais de saúde, em especial ao enfermeiro, complementar as análises sobre as perspectivas desse agravo, considerando a identificação dos grupos de maior vulnerabilidade e seus impactos sobre o potencial de vida produtiva dos indivíduos. Por essas razões, o estudo tem como objetivo realizar a análise epidemiológica de mortalidade por violência autoinfligida do Estado de Minas Gerais, por meio do cálculo do indicador Coeficiente de Mortalidade Padronizado (CMP) para $10^{5}$ e Anos Potenciais de Vida Perdidos (APVPs).

\section{METODOLOGIA}

Estudo ecológico, exploratório, do tipo série temporal sobre as características epidemiológicas de mortalidade por violência autoinfligida do Estado de Minas Gerais, Brasil, entre os anos de 1996 a 2007. O limite temporal proposto deve-se a disponibilidade de registros de óbitos nos Bancos de dados do Ministério da Saúde, correspondentes à $10^{\mathrm{a}}$ Revisão da Classificação Internacional de Doenças (CID-10), do Grande Grupo de Causas [(X60-X84)] - Lesões Auto-provocadas.

Os dados utilizados foram extraídos do Sistema de Informação em Mortalidade (SIM), publicados pelo DATASUS. ${ }^{13}$ As bases para as estimativas populacionais foram obtidas através das projeções inter-censitárias da Fundação Instituto Brasileiro de Geografia e Estatística (IBGE), obtidas na página da Internet. ${ }^{13}$

Para análise das séries históricas da violência autoinfligida foram considerados os triênios do período (1996-2007), e para o cálculo do Coeficiente 
de Mortalidade Padronizado (CMP) utilizaram-se as categorias: Coeficiente de Mortalidade Específico $\left(\mathrm{n}^{\mathrm{o}}\right.$ total de óbitos sobre a população $\left.\times 10^{5}\right)$, Coeficiente de Mortalidade por Sexo ( $\mathrm{n}^{\mathrm{o}}$ de óbitos de um dado sexo sobre a população do mesmo sexo $\times 10^{5}$ ) e Coeficiente de Mortalidade por Idade $\left(\mathrm{n}^{\mathrm{o}}\right.$ de óbitos de determinado grupo etário sobre a população deste grupo $\times 10^{5}$ ).

Para a obtenção dos APVPs, por óbitos por lesões autoinfligidas, foi feita a distribuição dos óbitos por agrupamento de idade. Em seguida, multiplicou-se o número de óbitos em cada intervalo e idade pelo número de anos que faltavam para atingir a idade limite de 80 anos. Essa diferença foi obtida a partir do ponto médio de cada faixa etária. A soma desses produtos possibilitou o cálculo de APVP, valor que representa o número estimado de perdas para uma causa específica. ${ }^{14-16}$ Adotou-se a idade limite de 80 anos por considerar que limites de idade mais elevada são mais inclusivos. Os óbitos iguais ou superiores à idade limite não foram considerados para o cálculo dos APVPs e atribui-se a essas mortes peso igual a zero. ${ }^{17-18}$

Para o cálculo do número médio de APVPs, que expressa a quantidade de anos que, em média, cada óbito por determinada causa subtraiu de uma pessoa (anos não-vividos), dividiu-se o número de APVP relativo pelo número de óbitos ocorridos por esse mesmo tipo de causa. Na análise dos dados foram empregados: número absoluto e número médio de APVP, idade na qual, em média, os óbitos ocorreram e o número de APVP por 100.000 habitantes, especificados segundo sexo.
A idade em que, em média, a morte ocorreu foi obtida subtraindo-se o número médio de anos não-vividos devido à determinada causa externa da idade máxima estipulada. O valor total de APVP foi obtido pelo somatório das APVP em cada grupo etário, aplicando-se a fórmula: APVP $=\Sigma$ ai $x$ di, onde: ai representa a diferença entre a idade limite e o ponto médio de cada grupo etário, pressupondo-se distribuição uniforme das mortes ocorridas em cada grupo; di é igual ao número de óbitos por uma causa específica neste mesmo grupo etário. Os APVP por 100.000 habitantes corresponderam aos indicadores acumulados para o triênio de análise, tendo como base a média das populações desse período.

O Projeto de Pesquisa denominado "Evolução Espaço Temporal dos óbitos por causas violentas em Minas Gerais", que produziu o banco de dados utilizados nesta pesquisa, incluídos os procedimentos do estudo, foi submetido ao Comitê de Ética em Pesquisa da Universidade Federal do Triângulo Mineiro, obtendo parecer favorável, sob o número 1651/2010.

\section{RESULTADOS}

No Estado de Minas Gerais (MG), no período de 1996 a 2007, ocorreram 123.986 óbitos por causas externas, sendo 7,6\% destes por lesões autoinfligidas (suicídio). Neste período, o Coeficiente de Mortalidade Padronizado (CMP) por 100.000 habitantes, por violência autoinfligida, foi de 4,29 casos/100.000 habitantes (Tabela 1).

Tabela 1 - Coeficiente de mortalidade por violência autoinfligida por 100.000 habitantes, em Minas Gerais, segundo sexo e faixa etária, de 1996 a 2007

\begin{tabular}{|c|c|c|c|c|c|c|c|c|c|c|}
\hline \multirow{3}{*}{ Indicadores } & \multicolumn{2}{|c|}{ 1996-1998 } & \multicolumn{2}{|c|}{ 1999-2001 } & \multicolumn{2}{|c|}{ 2002-2004 } & \multicolumn{2}{|c|}{ 2005-2007 } & \multicolumn{2}{|c|}{$1996-2007$} \\
\hline & Coef* & $\mathbf{n}$ & Coef & $\mathbf{n}$ & Coef & $\mathbf{n}$ & Coef & $\mathbf{n}$ & Coef & $\mathbf{n}$ \\
\hline & 3,61 & 1830 & 3,57 & 1910 & 4,77 & 2657 & 5,21 & 3044 & 4,29 & 9441 \\
\hline \multicolumn{11}{|l|}{ Sexo } \\
\hline Masculino & 5,88 & 1475 & 5,80 & 1534 & 7,63 & 2101 & 8,31 & 2403 & 6,90 & 7513 \\
\hline Feminino & 1,37 & 351 & 1,39 & 376 & 1,97 & 555 & 2,17 & 641 & 1,73 & 1923 \\
\hline \multicolumn{11}{|l|}{ Faixa Etária } \\
\hline 5 a 9 anos & - & - & - & - & - & - & 0,02 & 1 & - & 1 \\
\hline 10 a 14 anos & 0,36 & 20 & 0,29 & 16 & 0,47 & 26 & 0,41 & 22 & 0,38 & 84 \\
\hline 15 a 19 anos & 2,64 & 142 & 2,82 & 158 & 3,37 & 196 & 3,13 & 181 & 2,99 & 677 \\
\hline 20 a 29 anos & 6,02 & 524 & 5,15 & 478 & 7,03 & 682 & 6,87 & 712 & 6,27 & 2396 \\
\hline 30 a 39 anos & 5,54 & 427 & 5,82 & 473 & 7,30 & 618 & 7,78 & 685 & 6,61 & 2203 \\
\hline 40 a 49 anos & 5,61 & 308 & 5,86 & 363 & 7,32 & 485 & 9,07 & 653 & 6,97 & 1809 \\
\hline 50 a 59 anos & 5,13 & 183 & 5,46 & 220 & 8,26 & 351 & 9,02 & 425 & 6,97 & 1179 \\
\hline 60 a 69 anos & 5,07 & 126 & 4,21 & 114 & 6,00 & 172 & 6,95 & 215 & 5,56 & 627 \\
\hline 70 a 79 anos & 4,97 & 62 & 3,67 & 53 & 5,98 & 92 & 6,06 & 104 & 5,17 & 311 \\
\hline+80 anos & 4,44 & 22 & 5,19 & 30 & 5,13 & 32 & 6,03 & 43 & 5,19 & 127 \\
\hline
\end{tabular}


Ao se adotar os coeficientes estabelecidos pela Organização mundial da Saúde (OMS), para fins de análise: baixo - menor que 5/100.000 habitantes; médio - entre 5 e 15/100.000 habitantes, alto - entre 15 e 30/100.000 habitantes, e muito alto - acima de 30/100.000 habitantes ${ }^{1}$, o CMP por violência autoinfligida em Minas Gerais, no período em questão, manteve-se em níveis considerados baixos, e acompanha a média nacional dos estudos realizados em 2000, de 4 casos / 100.000 habitantes. ${ }^{19}$

Ao decompor o CMP por $10^{5}$ da violência autoinfligida em MG, por período, observou-se que de 1996 a 2004 (três triênios), manteve-se em níveis relativamente baixos, 3,61, 3,57 e 4,77. Já no triênio correspondente ao período de 2005 a 2007, o CMP por $10^{5}$ elevou-se para o nível médio - 5,21. Portanto, no período estudado, houve um progressivo aumento do CMP do primeiro triênio (de 1996-1998 - 3,61) para último triênio (de 20052007 - 5,21) (Tabela 1).

Em termos proporcionais, em Minas Gerais, houve aproximadamente quatro óbitos do sexo masculino para um feminino, por violência autoinfligida. No período em questão, houve um gradativo aumento do CMP em ambos os sexos. Dessa maneira, percebe-se que para o período de análise, o CMP foi quatro vezes maior entre os homens do que as mulheres. Vale ressaltar que o CMP dos homens, 2005 a 2007, foi de 8,31/100.000 habitantes. Esse indicador coloca a violência autoinfligida em homens, no nível intermediário (Tabela 1).

Em relação à média de idade do $\mathrm{CMP}$, do Estado de Minas Gerais, por 100 mil habitantes, observamos que eles apresentam maiores resultados para as faixas etárias entre 20 a 59 anos, sendo a média dos CMPs para os anos em análise, de 6,7/100.000 habitantes, classificando como um indicador de risco intermediário, para essas faixas etárias. Entre os triênios de análise, o último triênio apresenta os maiores indicadores, os quais se destacam para as faixas etárias de 40 a 49 anos e 50 a 59 anos, com coeficiente médio de 9,04 óbitos/100.000 habitantes, chamando a atenção para a vulnerabilidade desse grupo no acometimento de óbitos por violência autoinfligidas.

Embora os estudos sobre violência autoinfligida estejam mais focalizados em adolescentes, o presente estudo constatou que os óbitos por violência autoinfligida em Minas Gerais, no período de 1996 a 2007, concentram-se na faixa etária social e economicamente produtiva, dos 20 aos 59 anos. Este estudo, realizado pela análise de dados de fontes secundárias do Sistema de Informação de Mortalidade do SUS (SIM), apresenta perfil diferenciado, no estado de Minas Gerais, para os óbitos por violência autoinfligida, em que os maiores indicadores para esse tipo de óbito se encontram a nas faixas etárias de 20 a 59 anos, com maior concentração dos óbitos para as faixas etárias de 40 a 49 anos e 50 a 59 anos.

Consequentemente, ao analisar a distribuição dos APVPs, a média da idade de óbitos manteve-se em torno dos 40 anos (Tabela 2). Ao avaliar os APVPs totais, observou-se uma redução discreta dos APVPs, no decorrer do período estudado. Essa diminuição advém do fato de quanto maior a idade em que a pessoa cometeu a violência auto infligida, menor é o APVP.

Tabela 2 - Distribuição dos anos potenciais de vida perdidos por óbitos por lesões autoinfligidas em Minas Gerais, 1996 a 2007

\begin{tabular}{lcccc}
\hline Período & \multicolumn{3}{c}{ APVP } & $\begin{array}{c}\text { Idade média } \\
\text { do óbito } \\
\text { (anos) }\end{array}$ \\
\cline { 2 - 5 } & Médio & Total & $\mathbf{1 0 5}$ & \\
\hline 1996-1998 & & & & \\
Masculino & 42,20 & 61102,5 & 730,21 & 37,80 \\
Feminino & 42,62 & 14490,0 & 169,97 & 37,38 \\
Total & 42,31 & 75815,0 & 448,81 & 37,69 \\
1999-2001 & & & & \\
Masculino & 41,87 & 62932,5 & 715,49 & 38,13 \\
Feminino & 42,41 & 15777,5 & 175,78 & 37,59 \\
Total & 41,98 & 78710,0 & 442,90 & 38,02 \\
2002-2004 & & & & \\
Masculino & 40,81 & 84472,5 & 920,34 & 39,19 \\
Feminino & 42,86 & 23617,5 & 251,93 & 37,14 \\
Total & 41,23 & 108115,0 & 582,73 & 38,77 \\
2005-2007 & & & & \\
Masculino & 39,74 & 93795,0 & 973,54 & 40,26 \\
Feminino & 41,20 & 26285,0 & 267,01 & 38,80 \\
Total & 40,05 & 120080,0 & 616,47 & 39,95 \\
1996-2007 & & & & \\
Masculino & 40,96 & 302302,5 & 3361,12 & 39,04 \\
Feminino & 42,17 & 80170,0 & 873,32 & 37,83 \\
\hline Total & $\mathbf{4 1 , 2 1}$ & $\mathbf{3 8 2 7 2 0 , 0}$ & $\mathbf{2 1 0 5 , 8 7}$ & $\mathbf{3 8 , 7 9}$ \\
\hline
\end{tabular}

\section{DISCUSSÃO}

No Brasil, as mortes por violência autoinfligida incluem-se nas causas externas, segundo a $10^{\mathrm{a}}$ Revisão da Classificação Internacional de Doenças (CID-10). Nesta linha, por mais que a mortalidade por causas externas ocupe o segundo lugar no perfil da mortalidade geral, as mortes por violência 
autoinfligida diferem do cenário internacional, com menor prevalência quando comparada às taxas européias e norte-americanas. ${ }^{16} \mathrm{Na}$ década de 90 , a violência autoinfligida representou 5,6\% das mortes por causas externas e $0,6 \%$ do total de óbitos, indicando um crescimento de $7,8 \%$ no período e uma razão de quatro mortes por cem mil habitantes. ${ }^{5,19}$

Estudo realizado em 90 países estimou a taxa de violência autoinfligida entre jovens de 15 e 19 anos em 7,4/100 mil. As maiores taxas foram em Sri Lanka (46,5/100 mil), Lituânia (23,9/100 mil) e Rússia (23,6/100 mil). O Canadá ocupa a $15^{a}$ posição $\left(10,8 / 100\right.$ mil) e os Estados Unidos a $34^{\text {a }}(8,0 / 100$ mil). A estimativa brasileira foi de 4,2/100 mil e taxas mais elevadas entre os meninos (10,5/100 mil) do que entre as meninas $(4,1 / 100 \mathrm{mil}) \cdot{ }^{20}$

Ao resgatar estudos realizados nas décadas passadas, observa-se que, na Austrália, no período de 1990 a 1994, as taxas de mortalidade por violência autoinfligida em jovens de 15 a 24 anos foi 25,7 no sexo masculino e 5,1/100.000 habitantes, no feminino. ${ }^{21} \mathrm{O}$ Departamento de Saúde Pública de Massachusetts divulgou que, no período de 1996 a 1998, as taxas médias anuais foram mais elevadas em mulheres com idades entre 15 e 19 anos (148,3/100 mil habitantes) em relação às demais faixas etárias. ${ }^{19}$ De 1983 a 2003, o serviço sentinela holandês constatou que as mulheres entre 10 e 19 anos foram responsáveis pelos maiores índices de violência autoinfligida. ${ }^{22}$

Já os dados preliminares, divulgados pelo Núcleo de Atenção ao Suicídio da Secretaria Municipal de Saúde do Rio de Janeiro, no período de agosto de 2000 a agosto de 2003, de 11 unidades de saúde e dois hospitais gerais, constataram que $60 \%$ das notificações foram de pessoas com até 34 anos, sendo a maior incidência na faixa de 15 a 24 anos $(30,4 \%)$ e com percentuais semelhantes entre homens e mulheres. ${ }^{23}$

Em 2000, no Brasil, observou-se uma taxa mais elevada de internação por tentativa de suicídio nos homens (6,8/100.000 habitantes) do que nas mulheres (4,2/100.000 habitantes). Entretanto, o sexo feminino apresentou maior taxa de internação do que o masculino, nos grupos de 10 a 14 anos e 15 a 19 anos (2,7 e 6,8/100.000 habitantes, respectivamente) e nos homens de 10 a 14 anos (2,5/100.000 habitantes) e 15 a 19 anos (5,5/ 100.000 habitantes). ${ }^{19}$

Em Minas Gerais, de 1998 a 2003, o número total de internações via Sistema Único de Saúde (SUS), decorrentes de violência autoinfligida foi de 14.443 , das quais $55,4 \%$ pertenciam ao sexo mas- culino. A faixa etária de 10 a 19 anos representou $16,2 \%$ do total dessas internações. Da mesma forma notou-se que a taxa de internação em mulheres foi superior (5,8 e 19,9/100.000 habitantes entre 10 e 14 anos e 15 a 19 anos, respectivamente) a dos homens (4,0 e 12,4/100.000 habitantes). ${ }^{8}$

Quanto ao procedimento utilizado na violência autoinfligida, há estudos que indicam que na década de 1980, 75,5\% dos adolescentes de 10 a 17 anos empregaram o uso abusivo de drogas e $10,4 \%$ a laceração ou a perfuração. ${ }^{24}$ Há outros estudos mais recentes que relatam que a violência autoinfligida, praticada em $80 \%$ aproximadamente dos adolescentes, foi por ingestão de substâncias que provocavam intoxicações exógenas, seguida de corte nos pulsos. ${ }^{25}$ Em Minas Gerais, os registros do SUS indicam que as lesões decorrentes de objetos perfurocortantes foram a segunda causa de internação entre adolescentes por violência autoinfligida. $^{8}$

A auto-intoxicação tem sido constatada como procedimento mais utilizado em ambos os sexos, sobretudo pelas mulheres que utilizaram este método, três vezes mais que os homens. ${ }^{8} \mathrm{O}$ estudo realizado em Ribeirão Preto sobre os atendimentos hospitalares decorrentes de violência autoinfligida também constatou predomínio em mulheres $(77,8 \%)$, sendo a ingestão de medicamentos $(65,3 \%)$ e de substâncias químicas $(20,8 \%)$, os procedimentos mais empregados, e em ambos os sexos. ${ }^{26}$

Entre os anos de 1996 e 2002 o procedimento mais utilizado pelos homens foi enforcamento, estrangulamento e sufocamento $\square 1,1$ óbito/ 100.000 habitantes e por arma de fogo (0,6 óbito/ 100.000 habitantes). Entre as mulheres, os procedimentos foram enforcamento, estrangulamento, sufocamento e auto-intoxicação, com taxas iguais de 0,3 óbito/100.000 habitantes. ${ }^{8}$

Estudos que associam a tentativa de violência autoinfligida indicam maior prevalência de planejamento suicida entre jovens do sexo feminino. ${ }^{27}$ Por outro lado, o estudo realizado na Argentina, com adolescentes entre 15 a 24 anos, indicam que os homens apresentam maior risco de morte por violência autoinfligida do que as mulheres $(19,2$ e 5,5/ 100.000 habitantes, respectivamente)..$^{28}$

Há estudos que relatam que existe uma associação entre o sexo e o procedimento utilizado para violência autoinfligida. Os homens optam por procedimentos mais letais (enforcamento e tiro por arma de fogo) do que as mulheres. ${ }^{29}$ Esse resultado legitima outros estudos que constataram que as meninas planejam e tentam mais, porém os 
meninos optam por procedimentos que acabam concretizando a violência autoinfligida. ${ }^{30}$

Uma das prováveis causas da violência autoinfligida pode estar atrelada à questão da prevalência de depressão. ${ }^{31}$ A relação entre depressão e violência autoinfligida foi evidenciada nos estudos sobre adolescentes que se sentiam mais sozinhos e/ou tristes no qual houve maior prevalência de planejamento suicida do que aqueles sem esse tipo de pensamento. ${ }^{27}$

Quanto à mortalidade, em Minas Gerais, no período de 1980 a 2002, em estudo sobre óbitos na adolescência, ocorreram 25.060 óbitos por causas externas de adolescentes, entre 10 e 19 anos (uma mulher para cada três homens), sendo $4,8 \%$ destes decorrentes de violência autoinfligida. Ao longo dos anos, os homens pertencentes ao grupo de 15 a 19 anos, apresentaram riscos mais elevados de morte com taxa de mortalidade média de quase duas vezes maior que as mulheres $(3,5 \mathrm{e}$ 2,0/100.000 habitantes). Porém, as taxas de mortalidade para os grupos de 10 a 14 anos apresentaram mais oscilações entre os sexos. ${ }^{8}$

Enfim, a taxa de mortalidade por violência autoinfligida nas principais capitais brasileiras vem apresentando um crescimento de $35,3 \%$ entre jovens de 15 a 24 anos. A cidade de Belo Horizonte apresentou taxa de mortalidade de 4,3 no ano de 1979 e 6,8/ 100.000 habitantes em 1995; portanto, um crescimento de $58,1 \%$ no período estudado. ${ }^{32}$

Atualmente, os principais modelos explicativos para a violência autoinfligida retratam três dimensões: o aspecto sociológico, que o trata no contexto histórico e cultural; o aspecto psicológico que o considera como resultado de conflitos internos dos indivíduos; e o aspecto nosológico, em que esse problema é visto como enfermidade, havendo convergência nessas dimensões nos episódios identificados. 9,19, 33

Não há como negar a complexidade da violência autoinfligida e as dificuldades sociais para a abordagem explícita dessa temática, em decorrência da estigmatização dos sujeitos e familiares envolvidos nesse processo, uma vez que, de um modo geral, as pessoas tendem a negar essa atitude definida pelo contra-fluxo natural da vida. ${ }^{5,8,19,34}$

Essa realidade também é um desafio para a compreensão ou interpretação das mortes por violência autoinfligida, haja vista que as informações disponíveis são relativizadas uma vez que a ocorrência da violência autoinfligida pode ser mais elevada do que os dados notificados nas fontes oficiais. ${ }^{5,34}$
O comportamento suicida ocorre na maioria das vezes, como reflexo de conflitos internos, sentimentos de depressão e ansiedade que acompanham uma profunda reorganização física, psíquica e social que acontece com as pessoas. Além do mais, o estudo realizado na Argentina demonstra que apenas $10 \%$ dos pacientes tinham conhecimento adequado de como enfrentar o problema intrafamiliar. ${ }^{27}$ Ainda que não seja uma tarefa fácil determinar a intencionalidade de atitudes autodestrutivas, vale ressaltar o papel da família que, geralmente, é mais figura mais próxima à pessoa com comportamento suicida, e capaz de intervir de forma precoce, encaminhando-o para uma assistência especializada.

A violência apresenta-se no panorama internacional e nacional como uma questão social e de saúde pública. Além do mais, as pessoas vivenciam hoje, alterações socioeconômicas que podem resultar em uma expectativa de aumento substancial das taxas de mortalidade por violência autoinfligida. Trata-se de uma temática interdisciplinar, complexa que não bastará para o seu controle apenas a responsabilidade ética de cada indivíduo e requer, por conseguinte, uma efetiva mobilização dos diversos setores governamentais e da sociedade civil. ${ }^{35} \mathrm{Neste}$ contexto, registra-se ainda a importância das pesquisas epidemiológicas para compreensão mais ampliada da violência autoinfligida e que possa influenciar no desenvolvimento de estratégias de prevenção desse agravo e promoção da saúde. ${ }^{10,34}$

\section{CONCLUSÃO}

No Estado de Minas Gerais, no período de 1996 a 2007, ocorreram 123.986 óbitos por causas externas, sendo $7,6 \%$ destes por violência auto infligida. Entretanto, o coeficiente de mortalidade por esse agravo de 4,29 casos/100.000 habitantes, é considerado baixo, conforme padrões da OMS. A distribuição por sexo apresentou proporção de para cada quatro óbitos do sexo masculino um óbito feminino. Em números absolutos, as mortes por violência auto infligida se encontravam concentradas na faixa etária social e economicamente produtiva, dos 20 aos 59 anos; estando os maiores coeficientes na faixa etária de 40 a 59 anos.

Em relação à distribuição dos APVPs a média da idade dos óbitos mantiveram-se em torno dos 40 anos. Houve redução nos valores de APVP, ao se comparar os triênios. Esse fato se relaciona com o aumento da idade de ocorrência desses óbitos e cada vez mais a mortalidade atinge pessoas mais velhas. É preocupante, ainda, o fato dos 
resultados da violência autoinfligida apontarem para a perda potencial social do sujeito em idade adulta, em Minas Gerais.

Todos esses dados devem ser considerados como fatores preocupantes para a saúde pública, não somente pela perda do potencial social do sujeito que cometeu a violência autoinfligida, mas também, para se atentar aos determinantes do processo saúde doença em seu aspecto ampliado, que influenciam as pessoas de faixa etária mais elevada a serem acometidos pela violência autoinfligida. Nesse sentido, cabe aos profissionais de saúde, como a Enfermagem, sensibilizar-se para a importância desse agravo no contexto social atual, promovendo abordagens estratégicas à população vulnerável, a fim de limitar sua ocorrência.

\section{REFERÊNCIAS}

1. Krug EG, Dahlberg LL, Mercy JA, Zwi AB, Lozano R, editores. Relatório mundial sobre violência e saúde. Genebra: OMS; 2002.

2. Organización Mundial de la Salud. Prevención del suicidio: un instrumento para docentes y demás personal institucional. Genebra: OMS; 2001.

3. Gawryszewski VP, Koizumi MS, Mello-Jorge MHP. As causas externas no Brasil no ano 2000: comparando a mortalidade e a morbidade. Cad Saúde Pública. 2004 Jul-Ago; 20(4):995-1003.

4. Durkheim E. O suicídio. São Paulo (SP): Perspectiva; 1982.

5. Parente ACM, Soares RB, Araújo ARF, Cavalcante IS, Monteiro CFS. Caracterização dos casos de suicídio em uma capital do Nordeste Brasileiro. Rev Bras Enferm. 2007 Jul-Ago; 60(4):377-81.

6. Ponce JC, Andreuccetti G, Jesus MGS, Leyton V, Muñoz DR. Álcool em vítimas de suicídio em São Paulo. Rev Psiquiatr Clin. 2008; 35(suppl 1):13-6.

7. MeneghelSN, Victora CG, Faria NMX, Carvalho LA, Falk, JW. Características epidemiológicas do suicídio no RS. Rev Saúde Pública. 2004; 38(6):804-10.

8. Abasse MLF, Oliveira RC, Silva TC, Souza ER. Análise epidemiológica da morbimortalidade por suicídio entre adolescentes em Minas Gerais, Brasil. Cienc Saúde Coletiva. 2009 Mar-Abr; 14(2):407-16.

9. Movahedi, S. Weaponization of the body and politicization of death. Rev Latinoam Psicopat Fund. 2009 Mar; 12(1):71-98.

10. Rocha FF, Sousa KCA, Paulino N, Castro JO, Correa H. Suicídio em Belo Horizonte entre 2004 e 2006. Rev Bras Psiquiatr. 2007 Jun; 29(2):188-99.

11. Reichenheim ME, Werneck GL. Anos potenciais de vida perdidos no Rio de Janeiro, 1990. As mortes violentas em questão. Cad Saúde Publica. 1994 Nov; 10(suppl 1):188-98.
12. Heck RM. Percepção social sobre categorias de risco do suicídio entre colonos alemães do noroeste do Rio Grande do Sul. Texto Contexto Enferm. 2004 Out-Dez; 13(4):559-67

13. Ministério da Saúde (BR). DATASUS. Departamento de Informática do SUS [base de dados na internet]. Informações de Saúde. Sistema de Informações sobre Mortalidade. Brasília (DF); 2010 [acesso 2010 Out 15]. Disponível em: http//tabnet.datasus.gov.br

14. Arnold MW, Silva MA, Falbo Neto GH, Haimenis RP. Anos potenciais de vida perdidos por mulheres em idade fértil na cidade do Recife, Pernambuco, vítimas de morte por homicídio nos anos de 2001 e 2002. Rev Bras Saúde Mater Infant. 2007 Nov; 7(suppl. 1):23-8.

15. Santana VS, Araújo-Filho JB, Silva M, AlbuquerqueOliveira PR, Barbosa-Branco A, Nobre LCC. Mortalidade, anos potenciais de vida perdidos e incidência de acidentes de trabalho na Bahia, Brasil. Cad Saúde Pública. 2007 Nov; 23(11):2643-52.

16. Araújo EM, Costa MCN, Hogan VK, Mota ELA, Araújo TM, Oliveira NF. Diferenciais de raça/cor da pele em anos potenciais de vida perdidos por causas externas. Rev Saúde Pública. 2009; 43(3):405-12.

17. Cordeiro R, Olivencia P, Eduardo R, Cardoso CF, Cortez DB. Kakinami E, et al. Desigualdade de indicadores de mortalidade no sudeste do Brasil. Rev Saúde Publica. 1999; 33(6):593-601.

18. Peixoto HCG, Souza ML. O indicador anos potenciais de vida perdidos e a ordenação das causas de morte em Santa Catarina, 1995. Inf Epidemiol SUS. 1999 Mar; 8(1):17-25.

19. Minayo MCS. Suicídio: violência autoinfligida. In: Ministério da Saúde (BR). Secretaria de Vigilância em Saúde. Impacto da violência na saúde dos brasileiros. Brasília (DF): MS; 2005. p.205-34.

20. Wasserman D, Cheng Q, Jiang GX. Global suicide rates among young people aged 15-19. World Psychiatry. 2005 Jun; 4(2):114-20.

21. Cantor CH, Neulinger K, Leo DD. Australian suicide trends 1964-1997: youth and beyond? Med J Aust. 1999 Aug; 171(3):137-41.

22. Marquet RL, Bartelds AIM, Kerkhof AJFM, Schellevis FG, van der Zee J. The epidemiology of suicide and attempted suicide in Dutch general practice 19832003. BMC Fam Pract.2005 Nov; 4(6):45.

23. D’Oliveira CF. Atenção a jovens que tentam suicídio: é possível prevenir. In: Ministério da Saúde (BR). Violência faz mal à saúde. Brasília (DF): MS; 2004. p.177-84.

24. Andrus JK, Fleming DW, Heumann MA, Wassell JT, Hopkins DD, Gordon J. Surveillance of attempted suicide among adolescents in Oregon, 1988. AM J Public Health. 1991 Aug; 81(8):1067-9.

25. Bahls SC. Aspectos clínicos da depressão em crianças e adolescentes. J Pediatr. 2002 Set-Out; 78(5):359-66. 
26. Avanci RC. O adolescente que tenta suicídio: estudo epidemiológico em uma unidade de urgência [dissertação]. Ribeirão Preto (SP): Escola de Enfermagem de Ribeirão Preto da Universidade de São Paulo, Programa de Pós-Graduação em Enfermagem; 2004.

27. Gutiérrez MA, Fernández SGM, Jiménez MM, Gutiérrez JMA, Tamayo OD. Modificación de conocimientos sobre conducta suicida en adolescentes y adultos jóvenes con riesgo. Medisan. 2009 Ene-Feb; 13(1):1-6.

28. Basile HS. El suicidio de los adolescentes en Argentina. Alcmeon Rev Arg Clín Neuropsiquiátr. 2005 Set; 12(3):211-31.

29. Larraguibel M, González P, Martínez V, Valenzuela $R$. Factores de riego de la conducta suicida en niños y adolescentes. Rev Chil Pediatr. 2000 Jun; 71(3):1-13.

30. Marín-León L, Barros MBA. Mortes por suicídio: diferenças de gênero e nível socioeconômico. Rev Saúde Pública. 2003 Jun; 37(3):357-63.

31. Piccinelli M, Wilkinson G. Gender differences in depression: critical review. Br J Psychiatry. 2000 Dec; 177:486-92.

32. Souza ER, Minayo MCS, Malaquias JV. Suicide among young people in selected Brazilian State Capitals. Cad. Saúde Pública. 2002 Maio-Jun; 18(3):673-83.

33. Minayo MCS, CavalcanteFG, Souza ER. A eloquência do gesto final: suicídio em Itabira. Rio de Janeiro: Claves/Fiocruz, 2003.

34. Viana GN, Zenkner FM, Sakae MT, Escobar BT. Prevalência de suicídio no Sul do Brasil, 2001-2005. J Bras Psiquiatr. 2008 Jan; 57(1):38-43.

35. Schraiber LB, D'Oliveira AFPL, Couto MT. Violência e saúde: estudos científicos recentes. Rev Saúde Pública. 2006; 40(Esp):112-20. 